DOI: $10.5216 /$ racs.v6.65696

\title{
El cuidado de la madre tierra con las palabras sabias de los ancianos desde la cultura zapoteca y la educación de los niños del pueblo de Santa Cruz Yagavila.
}

\section{Wilfrido Emigdio Santiago Cruz ${ }^{1}$}

\section{RESUMEN}

En el presente escrito doy a conocer la importancia que tienen los ancianos en la transmisión de conocimientos para la educación y formación de los niños desde la familia, mismo que se debe tomar en cuenta, pero sobre todo visibilizarlos en las escuelas llámese "Educación Formal" y de Educación Indígena. En el marco de la investigación, la pertinencia del artículo está en confirmar que con la educación propia de los pueblos originarios puede darse el dialogo de saberes entre las aportaciones de la ciencia, con los conocimientos comunitarios, pero retomando y partiendo de los conocimientos de los ancianos y sus palabras sabias, con la finalidad y propósito de aportar elementos para una educación contextualizada y pertinente que requieren los niños y niñas de los pueblos originarios, se resalta también el papel que juega el docente para que estos conocimientos se enriquezcan y se potencialicen desde el ámbito educativo y pedagógico.

PALABRAS ClAVE: Discurso de ancianos. Palabras sabias. Educación. Filosofía propia. Yuuyaadzaa (tierra, monte, día), Bë'gracia dzaayela (espíritu-aire, día, noche).

\section{Yela rhapa chi'i ki xhnarhu yuuyaazaa, len xhtidzake bëni gula xhidza, len yela rzëëdikibedoo ki Yedzi Santa Cruz Yagavila.}

NABABIS

Bayuchhi rhun ugilay ubekirhu bixhnana rhenkine inake bëni gula kiero, kate tuilenke didza len yuuyaazaa kierhu, kete tuike xhkalen orna rhu una xhsei bidoo doo na tazee, kete ruziieke bedoo, kate rnabake lawi ki ka kelake gunke liichhike, len kate rhulidzake len rhuchizake bëë gracia ki bunachi nugan, tuni gulunke xhus xhtorho bëni xhidza guula kierho. Tunii rhuchhiaani rdixhjëa naka bena gunëzta nakaxh na tunke; lenka bëni gula doo len balakanu nigula doo ki lu yëdzi xhidza kierhu, bea'lenlake didza len gunabayuuchha nakaxh tunke tuliidzake yuuyaazaa kirhu. Lake gulana'ke, tuni tunke ki ka yuuyaazaa kierhu guna kierhu lataj kate inabarhu bi inabarhu lawi, guna kierhu lataj gazarhu bi gazarhu lawi, kate rhuirhu xhkalen ka, kate rhunabarhu len rhuchizarhu lacchidoo bunachi nuga'an. Kanaka tuni rhunrhu kika lei rhuna kierhu lataj tzagarhu lei chhia'. Lei naka la'ayaka' tuana rhuirhu ki bidodoo rhulaa' kierhu, len bidodoo rhuna kierhu lei len béni zeu luxhubaa. Lu gichi ni rdixhjëida tu naka ga gunke bëni usedi bedoo, nakala ilunke ului'ekene yela rhapachi'i yuuyaazaa kierhu kana rnake xhus xhtoorho, len xhtidzake bëni gula kiero, niaki tuana yela rnabayuchhini, rhun ga ugunirhu chhin yutee tu naka ga gun rheo kulen, nakin kuarhu tu tunke bëni gula kierhu ki ka ukanrhu lei kieka bedoo zaa talajbe,

${ }^{1}$ Universidade Nacional Autónoma de México (UNAM), Cidade do México, México. E-mail: wilmarstg@gmail.com. 
guirhu didza len lake, inabarhu len lake, kuarhu chi'ke ki kakebe nu gida isiyi' rheo cha chi bëni ni gulatike. Ni rhuzueja neda lu gichi ni yugutee nakaxh bena gunëzta tuni bulekanke kierhu xhus xhtorho.

Yela rdëxhjëi': Xhtidzake bëni gula, didza tzawi, yela nazëdi, yela rhajni'kii, yuuyaadzaa, bë'gracia, dzaayela.

Una biblioteca de sabidurías interminables tiende a cerrarse, cuando llega el momento en que un anciano (a) retorna a la madre tierra que lo vio nacer. (Wilfrido Emigdio Santiago Cruz)

\section{Introducción}

Para aterrizar al objetivo de alguna investigación es importante para nosotros los seres humanos reconocer el papel que juega nuestra experiencia en todo ámbito, ya que debido a ella hemos ido evolucionando en nuestra formación tanto emocional como social.

Las experiencias hechas enseñanzas y aprendizaje, se van adquiriendo en constante dialogo con las personas mayores que de alguna u otra forma han transmitido sus conocimientos que han adquirido mediante sus vivencias.

En este escrito hago mención sobre la sabiduría de los ancianos y la forma que se han vuelto sabios en las comunidades originarias. Sobre todo, la forma en como ellos transmiten sus conocimientos a las nuevas generaciones para el desarrollo de valores sólidos y bien cimentados para vivir en armonía y de forma más humana con nuestro mundo natural y cultural.

Para la transmisión de estos conocimientos la lengua ha jugado un papel muy importante, ya que desde épocas inmemoriales ha sido el medio por el cual se han transmitido, ya sea en forma oral o como lenguaje en forma escrita y que gracias a ello la vida cultural y natural de los pueblos siguen vigentes, sobre todo se ha mantenido la historicidad mediante la lengua. "La lengua es un producto social de la capacidad de expresarse y de comunicarse...al ser un producto social constituye un conjunto de convenciones y normas socialmente aceptadas que hacen posible la comunicación” (López, 1989:10). Mediante la lengua entonces se ha sabido educar y formar a las nuevas generaciones en el aprendizaje de valores, en específico el respeto a todo lo vivo y no vivo que existe en la madre tierra.

A pesar de la persistencia de la cultura eurocéntrica o del saber científico, la resistencia de nuestros pueblos ha prevalecido, no para quitarle el valor a la ciencia, sino para mantener su cultura y una de ellas la lengua que nos da identidad. La legua ha jugado un papel fundamental en el 
desarrollo de la humanidad, por lo que ha sido y sigue siendo el elemento por el cual se han mantenido vivas las demás manifestaciones culturales de nuestros pueblos y por el constante diálogo se han fortalecido, prevalecido y practicado los saberes que nos heredaron nuestros ancestros. Prueba de ello los discursos ceremoniales enfocados a la madre tierra para hacer pedimentos, agradecimiento, levantamiento de espíritu, llamamiento de corazón; desde la cultura zapoteca del pueblo de Santa Cruz Yagavila.

\section{Palabras sabias de los ancianos y su importancia en el desarrollo de valores, para una educación contextualizada.}

Las carreteras de terracería con su fragancia a tierra mojada por los meses de mayo, junio y julio, meses en donde las primeras aguas de lluvia visitan las labradas tierras de los campesinos, en donde las primeras germinaciones se van asomando para disfrutar de los interminables boscosos horizontes por un lapso de cinco meses.

Por las mañanas el azulado del humo empañan las tejas de las casas, y que a tempranas horas la fragancia del café recién hervido se mezcla momentáneamente con el aire al respirar, incitándonos a probar de ese exquisito café sorbo a sorbo.

Santa Cruz Yagavila se llama este pueblo, un pueblo de cultura zapoteca perteneciente al Distrito y Municipio de Ixtlán de Juárez, Oaxaca; se localiza a $17^{\circ} 26^{\prime}$ de latitud Norte y a $96^{\circ} 20^{\prime}$ longitud Este y a "a una altura sobre el nivel del mar de 1,062 metros. Comisión Geográfica Exploradora, 1909” (Pérez G. R, 1956:385). A medio declive de la montaña conocida en zapoteco gia'yaziuu y en castellano quiere decir cerro del trueno. Pueblo originario zapoteco que me vio nacer y crecer, pero que con el tiempo y por las necesidades de seguir adelante anhelando un mejor futuro y queriendo ayudar a mis padres, dejé para seguir en este transitar de la vida profesional.

Por los años 80 a 90 el máximo nivel educativo en Yagavila, era el nivel primaria de educación indígena, razón y motivo que orilló a mi padres a tomar la decisión de mandarme a recibir mis estudios en otros pueblos de la sierra, teniendo siempre en mente, de que la escuela era un espacio donde el sujeto puede cambiar su vida y su conocimiento para provenir un mejor futuro, desde sembrar un grano de maíz, hasta ir a la escuela para tener un mejor trabajo que le proporcione medios para la subsistencia.

R. Articul.const.saber, 2021, v.6: e65696 
Siendo así e impulsado en busca de un mejor futuro, llegué a otro pueblo serrano y zapoteco llamado San Mateo Capulalpam de Méndez, perteneciente al mismo Distrito de Ixtlán de Juárez, en este pueblo recibí mis estudios de nivel secundaria y de bachillerato.

Capulalpam de Méndez, un pueblo totalmente diferente al mío, donde la gente era el 98\% monolingües en Castellano, tanto, que para mis compañeros de la escuela era como una burla que yo hablara mi lengua originaria, al usar huaraches en vez de zapatos; me llamaron rinconero, huarachudo, cafetero, ésta última palabra lo decían con la idea que tenían de que, en el pueblo, de donde yo salí, no se conocía la leche. En palabras mayores en el salón se daba la "IGNOMINIA"2.

Realmente no eran culpables, no, porque desde el seno familiar y desde la educación que recibieron sus padres las ideas y los conocimientos eran ya homogenizados. Más aún porque ya se consideraban como personas urbanizadas por la cercanía que había del pueblo a la ciudad de Oaxaca. También las nuevas generaciones ya tenían la ideología de que, solo el que hablaba el español y el que usaba zapatos y vestía con trajes elegantes, era el que triunfaba y que podía mejorar su condición de vida, pero esta idea es debido a la formación del estado; como dice Lois Meyer en una entrevista con Noam Chomski "la formación del estado, casi siempre por la fuerza, ha tratado de imponer estándares educativos nacionales con el fin de transformar a la gente en individuos similares" (Meyer y Mandonado, 2011:44).

El objetivo que persigue la educación hegemónica hoy en día en un cierto porcentaje va en decadencia, ya que gracias a un largo proceso de concientización que ha habido en nuestros pueblos y por nuestros pueblos por parte de las personas que se han interesado en darle un sentido contrahegemónico al monoculturalismo epistémico, se va trazando un sendero educativo diferente, donde se retoma los saberes locales, los saberes vivenciales, la vida de nuestros pueblos; se parte del contexto cultural de nuestros pueblos originarios y de sus vivencias.

En cuanto a la gente adulta, fueron muy respetuosos con mi cultura, algunos decían que querían hablar como yo, me ayudaron igual a fortalecer mi identidad, me decían que no debía hacerles caso a los niños del pueblo, ya que ellos desconocen el valor que tiene la lengua como cultura, hablo específicamente de las personas de 50, 60 hasta 70 años.

Específicamente de un profesor de 70 años que respondió el nombre de Eliseo Luna (tío Cheo) (+) un profesor jubilado que me rentó su casa para vivir durante mi trayecto como alumno de la secundaria y bachillerato, por la mañana todo los sábados y domingos me despertaba para

\footnotetext{
${ }^{2}$ Sustantivo (f) Ofensa grave que afecta la dignidad o el honor de una persona. (https://diccionario.reverso.net)
} 
regar sus plantas y para sembrar algunos frutales y hortalizas en su terreno al lado de don Mache, un anciano de unos 60 a 65 años en ese entonces; recuerdo sus palabras llenas de sabiduría que me decía cuando regaba, “alimenta las plantas son seres como tú, cuídalas y abónalas, también tienen hambre como tú, tu papá te habrá dicho algún día que la tierra es también nuestra madre porque nos alimenta"3. Deberas hijo eso me decía mi padre cuando yo era niño.

Después de regar y labrar la tierra, durante el desayuno que preparaba doña Besadigna más o menos de la edad de 57 años del mismo pueblo, acompañado de Don Miguel Luna anciano de Capulalpam de alrededor de 68 años de edad; tío Cheo me decía "ya vez hijo, ya vez que la tierra nos alimenta, ¿de dónde crees que viene el pan, de donde crees que vienen las verduras?, pues de la tierra muchacho, por eso te decía cuídala, así como se cuida a una madre"4. En cuanto a tu zapoteco no debes avergonzarte, háblalo donde quiera que vayas eso te ayudará a ser mejor que los niños de mi pueblo y ya con el tiempo irás aprendiendo el castellano. Ante las palabras de aliento de tío Cheo seguí con mis sueños, el de salir adelante en busca de un mejor futuro.

Estas palabras me motivaron a seguir comunicándome y dialogando en mi lengua originaria, que es el zapoteco mi lengua materna. A la vez empecé a reafirmar las palabras que decía mi padre cuando labrábamos la tierra. Con esta forma de pensar seguí estudiando hasta terminar el Bachillerato.

Seguro estoy de que ser de un pueblo originario es un orgullo, ya que la educación que transmiten los padres a los hijos, emergen conocimientos vivenciales, estos conocimientos no viene plasmados en alguna revista o en el plan y programa educativo. “¿Dónde está el conocimiento de la cultura campesina? No está en los libros de texto, está en la cabeza de las mujeres que la enseñan a sus hijas y estas últimas a sus hijas; así va de generación en generación y se enriquece y se hace más complejo" (Meyer y Maldonado, 2011, p.57).

Muy importante se hace entender y comprender que los pueblos originarios poseen saberes que van reconstruyendo nuestros valores culturales, sobre todo la formación que se reciben de los padres, lo abuelos, los tíos, etc., todas van reforzando la relación que se tiene con la madre tierra, partir de ella como elemento central para educarnos. Fortaleciendo de esta forma nuestra cultura como un esfuerzo que se ha mostrado para su revalorización. En tanto que:

\footnotetext{
${ }^{3}$ Palabras de Don Maximiliano Cosmes (Don Mache) de Capulalpam de Méndez.

${ }^{4}$ Comentarios de tio cheo (+) Profr. Jubilado de 70 años de edad. De Capulalpam de Méndez.
} 
...los esfuerzos que invierten los pueblos indígenas en la educación de sus niños y jóvenes, así como en la recuperación de valores culturales, no tiene otro fin que éste. La libre determinación de los pueblos indígenas, a diferencia de los viejos procesos de formación del estado nacional, persiguen los caminos de la reconstrucción de valores humanos universales y el reconocimiento de la tierra como la casa grande para todos (Meyer y Maldonado, 2011, p. 86).

Al hablar de cultura hago referencia en la forma propia de ver el mundo, en sus formas de vivir y de concebir la vida, pero que debido al sistema educacional han sufrido grandes modificaciones, mismas que tienen la intención de universalizar y homogenizar a todo individuo exterminando parte de la misma cultura propia.

El sistema educativo tiene el objetivo de "nivelar" o de homogenizar a las personas, destruyendo en consecuencia lo que llama la "cultura individual", asumida como compleja y basada en tradiciones propias...El sistema educativo, altamente estratificado, coloca a las personas en roles especiales específicos, la cual requiere la eliminación de las diferencias individuales, culturales, de identidad grupal o lingüística (Meyer y Maldonado, 2011, p. 284).

Éste es el contexto actual en el que se ubica y atraviesa la educación en México, en específico la educación indígena, como oficialmente se le nombra, un sistema educativo que históricamente se ha subordinado a los planteamientos de la educación nacional.

Durante el bachillerato y siguiendo una ideología homogénea, me mandaron a prácticas en pueblos originarios como: San miguel Abejones, Yuvila, el Punto entre otros, para llevar acabo mi servicio social como técnico agropecuario, este servicio consistía en el trazo de curvas de nivel y siembra de barreras vivas para evitar la erosión del suelo, durante estas prácticas me di cuenta de la importancia que tenía en la forma de vivir de estos pueblos, sobre todo la relación que mantenían con la madre tierra. Para mí, fue un saber que nunca me enseñaron en la escuela y que tampoco lo podía encontrar en algún libro en ese entonces.

Estas prácticas que realizaba fueron el punto de partida para retornar a mi pueblo, pero solo en periodos vacacionales con el objetivo de ver si era igual, similar o diferente en cuanto a cultura y creencias con los otros pueblos.

Al regresar a mi pueblo me di cuenta que no era lo mismo que vivir y convivir ahí, las cosas fueron cambiando y conforme iba avanzando en mis estudios me fui perdiendo de grandes aprendizajes, con mis padres, los ancianos, también de los padrinos que juegan un papel fundamental en la formación y educación de los niños, por lo que los padrinos deben ser personas 
adultas, que sepan conducir a los padres jóvenes que por alguna u otra razón carecen de experiencias para educar y formas a sus hijos.

Desde siempre en este pueblo, a los ancianos y ancianas se les guarda mucho respeto por sus sabias palabras o consejos que conducen a vivir en completa armonía con todo lo que nos rodea, en si nos enseñan a vivir con más sentido humano, en donde reina la hermandad.

En las asambleas comunitarias estas sabidurías resaltan y se viven, cuando se acuerda que mediante trabajos comunitarios como el tequio, el pueblo emprende trabajos de limpieza, cuidado y protección de sus recursos naturales, como: el cuidado y limpieza de los mantos acuíferos, limpia de veredas, limpia de brechas colindantes, abonamiento y siembra de plantas en zonas deforestadas, etc., en sí, el pueblo debía mantenerse limpio desde las colindancias con otros pueblos, hasta los patios de tierra que tenían las casitas de adobe, todo gira en torno a una armonía y con aprendizajes muy sólidos en cuanto a cómo vivir la vida en el pueblo.

Por lo tanto: las aspiraciones, los sueños y las propuestas de los pueblos indígenas no son solamente para nosotros sino también son alternativas a nuestro entender para resolver los problemas de la humanidad, por lo que la resistencia debe convertirse en una convergencia real desde todos los sectores conscientes y comprometidos con los seres humanos, la madre naturaleza y la vida (Meyer y Maldonado, 2011, p. 207).

Esta forma de adquirir conocimientos en nuestros pueblos, está basada en los problemas reales de la cotidianidad, en donde uno como alumno de la vida y no de la escuela aprendemos a desenvolvernos con la capacidad de resolver problemas que se viven en nuestro entorno y no solo quedarse en la adquisición de saberes como pretende el sistema educativo nacional.

Durante los tequios o trabajos comunitarios la gente aprovechaba en dialogar, contando anécdotas, experiencias, echando un poco de humor, narrando cuentos; los mayores dando consejos a los más jóvenes.

Cuando los trabajos se realizan fuera del pueblo estas pláticas se llevan a cabo en el transcurso del camino, en la hora del almuerzo y en la hora de comida, porque durante el desarrollo de algún trabajo se hace con mucho respeto y concentración, para evitar así accidentes que afecten a algún ciudadano de la comunidad, ya que ahí todos se cuidan y se llaman "BËCHA",5.

"BËCHA"” palabra zapoteca que en castellano significa hermano; en Santa Cruz Yagavila todos los habitantes nos llamamos hermanos, al igual llamamos a los que conforman el sector

\footnotetext{
${ }^{5}$ Palabra zapoteca de Santa Cruz Yagavila, que significa hermano.
} 
rincón, los nueve pueblos zapotecos y lo decimos asi: yuguterhu nakarhu bëchi luza'rhu', que en castellano quiere decir (todos somos hermanos), esta palabra se ocupa para nombrar a todo ciudadano de los pueblos en cualquier lugar y en todo momento. Y es una de tantas que nos dejaron nuestros ancestros.

Entonces se considera que mediante las palabras sabias los ancianos transmiten conocimientos que dan armonía y tranquilidad y forma con prudencia el vivir de las nuevas generaciones, vislumbrado un futuro diferente a la que se ha estado viviendo y lo que está por vivirse.

En Santa Cruz Yagavila los ancianos son los poseedores de sabidurías en cuanto al desarrollo de valores, ya que son los que han servido al pueblo con los cargos comunitarios tanto municipales, religiosos y comunales, han estado como sabios en la escuela de la vida en donde desarrollan valores como: El respeto a los mayores y entre hermanos, el cuidado y la protección de nuestra madre naturaleza, el amor a los padres; podemos decir que ellos forman la primera modalidad educativa desde el núcleo básico de la sociedad. Llegan a ser sabios del pueblo por la experiencia que han vivido y mediante los cargos municipales, religiosos y comunales que han cumplido. En el momento que se empieza a adquirir un rango de respeto y una jerarquía entre ciudadanos es cuando se hayan cumplido los cargos desde policía, mayor de vara, hasta regidores, es en este momento donde se van adquiriendo ciertos conocimientos que con el tiempo vuelve sabio al sujeto.

Una vez cumplido cabalmente con estos cargos entonces son acreedores a esperar un cargo más alto jerárquicamente, y éste es el de Agente Municipal o Síndico Municipal, para posteriormente y por ley comunitaria fungir como Alcalde Constitucional, con este último cargo cumplido pasan a formar el consejo de ancianos. Este grupo lo conforman los ancianos que han servido al pueblo y esto los hace ser ancianos sabios de la comunidad aun y a pesar de que no hayan recibido alguna instrucción escolar.

Por otro lado, también hay otro grupo de personas que se les llama caracterizados, estas personas que conforman dicho grupo la mayoría han estudiado desde la primaria hasta un nivel superior, ellos forman parte de la organización política del pueblo y que junto a los sabios tratan asuntos relacionados a las problemáticas del mismo, buscando solución o apoyo para algún beneficio común. Entre ellos hay maestros de danza, música, artesanos, profesionistas, etc. 
A pesar de la posición económica de cada individuo, cuando se trata de servir al pueblo con los servicios comunitarios se tiene que cumplir porque es una ley que ha quedado desde tiempos inmemoriales, todo esto se hace sin remuneración alguna, ya que son servicios comunitarios y obligación de los ciudadanos. Y desde esta forma de vida van adquiriendo nuevos conocimientos, aparte de la que han adquirido dentro de la familia y que lo van trasmitiendo a las nuevas generaciones, esto con la finalidad de que cuando lleguen a una edad adulta sepan cómo cumplir con lo que el pueblo les encomiende. Es una propia modalidad educativa donde desarrollan sus diferentes capacidades como seres humanos. Ruiz argumenta que:

\footnotetext{
La primera modalidad educativa practicada por los aztecas tenía lugar en el seno familiar, comenzaba desde el día de nacimiento y se desarrollaba los primeros años de vida bajo el cuidado de los padres en la intimidad familiar ... semejantes cuidados se tomaban en la educación del aspecto moral y de la buena manera de comportarse, transmitiéndoles diversas normas de vida para el seguimiento de un camino recto repleto de altas concepciones éticas (Ruiz, 2013, p.271).
}

Hoy en día estos saberes aún siguen vigentes debido a la resistencia que siempre se ha mostrado y que ha durado, fortaleciendo y refrendado la identidad, haciendo que la vida comunal sea la máxima expresión en una gama de diversas manifestaciones en diversos ámbitos: lingüístico, gastronómico, artístico, filosófico y cosmogónico, estos forman la filosofía propia de los pueblos originarios, la filosofía es "la base de cualquier civilización. La estructura fundamental de la sociedad se encuentra en una compleja explicación, que le da significado y sentido a la vida y al mundo" (Marín G. 2010:107).

En el 2007, una emoción y alegría inmensa sentí el primer día que ingresé para emprender la aventura de ejercer la docencia, más aún al saber que trabajaría en los pueblos originarios de la cultura zapoteca de la Sierra Juárez, cultura que me vio nacer y crecer. A pesar de las políticas del gremio magisterial, había algo muy profundo que me motivaba, este era el de convivir con la gente de los pueblos, algo tan puro que me hacía retornar a mi infancia con mi forma de vivir, convivir y aprender.

Recorrer cada una de las curvas que conducen a nuestra región para llegar a los pueblos era algo incomparable sin precio alguno, es mas era como adentrarse al paraíso propio del saber y como sendero por transitar era la Sierra Juárez; en el transcurso, hacer descansos y disfrutar de las diferentes estaciones del año, como por ejemplo: en otoño mirar como los arboles van cubriendo 
de un aterciopelado amarillo los caminos, caminos que al ritmo y al silbido del viento se desnudaban totalmente para el deleite de las pupilas.

En invierno sentir la frescura y el frio del viento besándonos la mejilla y el resto del cuerpo, anunciando a la vez un año más que está por terminar y otro por comenzar, invitándonos a disfrutar del rico café de olla serrana, con su exquisito pan de trigo. Pero su objetivo, no solo es eso, sino de hacernos saber sobre la importancia que juega dentro de nuestra habitad el calor de nuestro señor sol, al darnos abrigo estrechándonos con sus fuertes abrazos hechos radiantes e aluminosos rayos, que van penetrando lentamente también en la madre tierra, para darle calor a los otros seres vivientes.

Siguiendo con este recorrido y después del abrazo de nuestro padre y señor sol, su calor va anunciando también la llegada de un nuevo ser que es la primavera con sus aves y flores multicolores. Todo sigue siendo alegría y felicidad pero en otro contexto y dimensión natural, un contexto que alegra la vida de los que en la madre naturaleza habitamos, un paisaje más que disfrutar, un momento más que vivir para seguir recorriendo las interminables curvas que nos conducen a los pueblos donde se encuentran aquellos inocentes seres ansiosos de querer saber, aprender y conocer más acerca del mundo que los rodea, seres que deben, quieren y anhelan un mejor futuro tanto personal como para su pueblo, aquellos que necesitan ser emancipados del yugo epistémico eurocéntrico como los niños y las niñas del pueblo de Santa Cruz Yagavila.

Para lógralo nosotros como docentes debemos ser la punta de lanza en demostrar que se puede dar un diálogo de saberes entre los conocimientos occidentales y comunitarios, para que de esta manera se dé la complementariedad de ambos. Para así no seguir el objetivo de la pedagogía moderna. Rengifo (2003) dice:

Los profesores rurales no encuentran una salida pedagógica para tal dificultad, a pesar de los esfuerzos que muchos de ellos realizan. Han sido preparados en una pedagogía moderna con un concepto de niñez y de desarrollo mental que no conversa con las concepciones y la realidad cognoscitiva de la niñez de las comunidades (Rengifo, 2003, p.22).

En mi trayecto como docente en el medio indígena, me percaté de que los pueblos conservan su filosofía y que los niños son portadores de conocimientos, solo que debido a la pedagogía hegemónica estos conocimientos están opacados en las escuelas, más en el de educación indígena. 
Desconsolado como docente me fue complicado retomarlos y trabajarlos en las aulas, razón misma que me impulsó a plantear y a estructurar un proyecto de investigación del cual me postulé en el posgrado en el año 2020 en la UNAM, en el área de pedagogía en su líneas de investigación antropología cultural y educación, y su campo de conocimiento de educación y diversidad cultural, el proyecto de investigación se titula "palabras sabias de los ancianos zapotecos de santa cruz yagavila (bunitiloo ben xhënlachhu), para el cuidado de la naturaleza: aportes para una educación contextualizada”. Esta investigación tiene como punto de partida visibilizar, enriquecer y potencializar los conocimientos que adquieren los niños desde el seno familiar mediante los discursos de los abuelos o sabios de la comunidad, esto con una mirada crítica ante lo impuesto por el Sistema Educativo Nacional.

Mostrar y demostrar también, que sí es posible lograr una educación contextualizada encaminada hacia la interculturalidad critica (Walsh 2008), que dé sustentabilidad a la diversidad cultural y natural, con ello se estaría logrando que la educación sea una educación comunitaria que fortalezca la diversidad y con una esencia liberadora. Para logarlo debemos implementar y practicar el término de la liberación desde la educación "plantear tan solo la posibilidad de la trasformación del mundo por la acción del pueblo mismo, liberado a través de esa educación, y anunciar así las posibilidades de una nueva y autentica sociedad es convulsionar el orden anacrónico en el que todavía nos vemos" (Paulo F., 1997:7), interactuando en la sociedad donde se dé un dialogo de saberes "Aprender a construir una nueva relación social y productiva y un diálogo con el otro" (Leff E. 2004:15), en donde un solo saber no se sobrepone al otro.

En este sentido las palabras sabias de los ancianos pasan a formar parte del desarrollo del conocimiento de los niños en cuanto a vivir en armonía, con respeto y con hermandad, ésta forma de vivir no solo se enfoca entre humanos, sino va directo a la relación espiritual con que se tiene con la madre tierra. Y estas han colaborado en el cuidado y protección de nuestra madre naturaleza. Fortaleciendo la relación que existe entre el humano con lo vivo y no vivo.

De esta forma se dan a conocer las palabras sabias que están inmersas dentro de los discursos de los ancianos de Santa Cruz Yagavila y que "se les ha considerado tradicionalmente parte de la prosa didáctica, documentos de instrucción con que los antiguos mexicas educaban a sus hijos en la buena conducta moral y la práctica de las fórmulas sociales” (Ruiz. 2013:270). Pero estas palabras de ancianos de Yagavila, no solo se centra en formar a los niños y jóvenes para una reacción humana o para una buena moral, sino va más allá del respeto desde lo disciplinario de la 
escuela; son palabras que poseen y dan sabiduría para vivir en armonía con todo lo vivo y lo no vivo en nuestra madre naturaleza.

Dentro del discurso de ancianos, éstas son las siguientes palabras que dentro de una triada forma una sola que es: yuuyaadzaa. Palabra zapoteca que se refiere a lo vivo, y hace mención a la tierra, monte y día, usándose para ofrendar a la tierra. Y las otras que son "Bunitiloo-Ben Xhën Lachhu" que literalmente se interpreta como "pierde de vista y haz grande tu corazón".
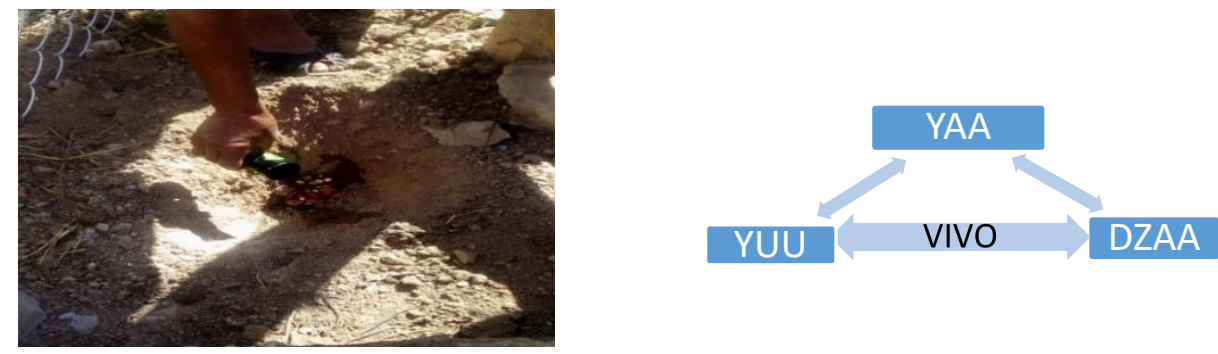

Ofrenda a la madre tierra para levantar el corazón quedado.

En los diversos acontecimientos como: Dar gracias después de la cosecha, pedir permiso para construir, labrar la tierra, levantamiento de espíritus, etc.

Y por otro lado hay otra triada de palabras sabias que forman una sola y que se refiere a lo no vivo, estas son: bë'gracia dzayela. Y se refiere al espíritu (aire), día y noche. Y se usan para llamar espíritus, levantar el corazón quedado en la tierra después de una caída y al curar de espanto.
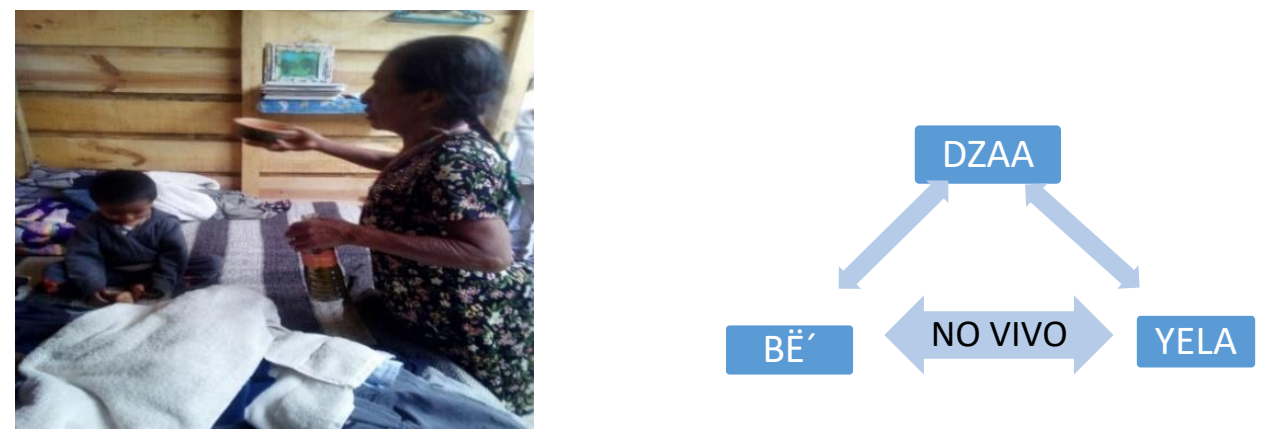

Llamamiento del espíritu de un niño.

Es necesario entonces investigar el papel que juega la escuela en la enseñanza de los valores universales y cómo se complementan con los conocimientos comunitarios estableciendo un dialogo entre estos saberes, por lo que "El diálogo en la educación permite compartir las ideas de unos con otros y lleva a la socialización; contrario a la individualización en el aprendizaje, muy propia de la educación tradicional o «educación bancaria», que es individualista”(Ocampo L.J.,2008:66), ya 
que son los espacios ideales donde se puede partir para fortalecer, rescatar, visibilizar, reproducir, difundir y potencializar estos conocimientos. Y que desde estos espacios se llegue a la formación de sujetos con valores bien cimentados, con capacidad de relacionarse e interactuar para construir una sociedad justa, armónica e igualitaria. Ya que de esta forma lo cultural transciende hacia el beneficio propio hasta el beneficio común del ser humano.

Se entiende que no se pretende aferrarse a nuestros conocimientos originarios a darlos por únicos y reales como lo hace la ciencia, sino lograr la aceptación de ambos. De esta manera el alumno vaya trascendiendo en su formación en los otros niveles educativos, pero partiendo desde su contexto cultural y natural.

Ante ello, el objetivo de este proyecto de investigación es analizar las palabras sabias de los ancianos de Santa Cruz Yagavila y su influencia en el aprendizaje basado en valores, en los espacios escolares, para aportar elementos a la educación contextualizada que requieren nuestros pueblos originarios. Ya que hoy en día es preocupante saber que se van perdiendo muchos valores; se le llama valores desde la epistemología eurocéntrica a los cuidados que se le da al medio ambiente, al protegerlas de la contaminación en sus tres tipos y a su aprovechamiento y uso irracional.

Desde los conocimientos de nuestros ancianos o de nuestros pueblos originarios se le llama relación espiritual con nuestra madre naturaleza, de esta manera el termino relación espiritual hace referencia a la protección, al cuidado, al diálogo que se establece entre el ser humano con la madre tierra que es un ser vivo que siente y que nos alimenta a los que en ella habitamos. Es en el diálogo donde emergen los conocimientos y las sabidurías de los ancianos, donde piden incentivar a nuestra madre, mediante pedimentos, ritos, oraciones, movimientos, sonidos etc. Acciones que están relacionadas directamente en su protección y cuidado.

Estas sabidurías donde están inmersas las palabras sabias se desarrollan en diferentes contextos y acciones tales como: la pedida de mano, en la siembra, en la cosecha, en la construcción de edificios comunales o casas para habitar, en el levantamiento de corazón, en el llamamiento del espíritu, en el nombramiento de las autoridades municipales y comunales. Son los espacios de donde los niños y los jóvenes aprenden, presenciando dichas acciones emprendidas por los ancianos y ciudadanos de la comunidad.

A mí me ha servido para reflexionar y de acuerdo a las vivencias que tuve al lado de mi padre cuando se tenía que labrar la tierra, más aún cuando él me llevaba en las diferentes actividades 
sociales y culturales de la comunidad, escuchaba los discursos que decían los ancianos. Esta forma de valorar y respetar a lo vivo y no vivo se aprende viendo, viviendo y haciendo, todo mediante la oralidad.

\section{La escuela como espacio para recuperar y potencializar la sabiduría ancestral de nuestras culturas originarias.}

He aquí el motivo del cual presento este texto para que usted lector se incite a seguir haciendo conciencia y trazar una nueva ruta para la educación de los niños de los pueblos originarios, tomando en cuenta que la columna vertebral en la formación de los niños son la familia, en especial los abuelos, los padres y el pueblo, ya que desde ahí se adquieren conocimientos vivenciales, significativas y de manera práctica. También mediante este se propone que los profesores visibilicen estos conocimientos que poseen los niños. A juicio de Bertely dice:

De esta manera están enseñando a los alumnos a reconocer que no siempre el maestro tiene la respuesta a un problema y que, en esos casos, se puede recurrir a otras personas que pueden saber más, entre los que pueden los padres. Con esta intervención el maestro enseña que el conocimiento no solo está en la escuela sino también fuera de ella (Bertely, 2000, p.13).

Si bien es cierto en Yagavila, los aprendizajes que poseen los niños las han aprendido de los padres y los padres de los abuelos, y, a lo que se quiere llegar es, que la escuela sea un espacio en donde se visibilicen y se potencialicen dichos conocimientos. Ya que la escuela como se comenta en párrafos anteriores es el espacio ideal para formar seres críticos, espacio en donde se les proporciona las herramientas al niño o a la niña para que vaya construyendo su propio conocimiento y que lo aplique en su vida diaria; en síntesis, invertir el papel que ha jugado la escuela, solo de esta forma podremos liberarnos del pensamiento impuesto por el occidente desde su sistema educativo. Encaminando todo lo expuesto hacia una pedagogía critica. Como dice Ortega (2009):

Consideramos que las orientaciones que ofrece la pedagogía crítica permiten promover el desarrollo de la autonomía, la participación, el reconocimiento y el respeto por la alteridad, la generación de espacios para la comprensión y la tramitación de los conflictos y la creación de ambientes sociales y comunitarios para reconocernos en apuestas colectivas (Ortega, 2009, p. 28). 
Por lo tanto, como profesor investigador propongo que desde las aulas y con la guía de los docentes se den enseñanzas más vivenciales, tales como: antes de iniciar cualquier actividad dentro y fuera del área escolar, los docentes y bajo criterio propio formulen discursos en donde resalten las palabras sabias de los ancianos tales como: yuuyaadzaa, bë’gracias dzaayela, Bunitiloo-Ben Xhën Lachhu, ya que son las palabras sabias que están inmersas dentro de los discursos de los ancianos, se les llama palabras sabias porque provienen de los sabios del pueblo, estas personas se hicieron sabias de acuerdo a la experiencia que han adquirido en su cotidianidad y a la vez al cumplir los cargos comunitarios que el pueblo les ha conferido dentro de sus discursos donde piden permiso a la madre tierra para construir un casa, abrir camino, levantar espíritus, para labrar la tierra y para levantar la cosecha, está inmersa la triada de palabras sabias, se les llama sabias porque, transmiten armonía, tranquilidad, felicidad, hermandad y seguridad profunda en la relación espiritual que tiene el hombre con la madre tierra exterminando de esta forma al "patriarcado" (Gustavo Esteba, junio 2020) se debe luchar en contra del mismo, para suprimir la jerarquía contra el saber hacer de los pueblos.

Tomando en cuenta que la educación desarrolla de manera práctica las capacidades intelectuales del individuo, lo moral y lo afectivo y que depende del hombre para transmitir conocimiento desde su cultura, entonces es importante recalcar que los discursos de los ancianos con las palabras sabias inmersas, dan conocimientos a los niños desde su propia cultura y desde la familia. Entonces estas palabras y los discursos se vuelven educativos desde el momento en que los niños se los apropian y los llevan a la práctica, de la misma manera para que estos discursos se vuelvan pedagógico.

El papel que se juega como docente es buscar los mecanismos necesarios, las estrategias y técnicas adecuadas para su aplicación en el proceso de enseñanza-aprendizaje vs aprendizajeenseñanza objetivo que persigue la pedagogía.

Un ejemplo como propuesta para incorporar las palabras sabias de los ancianos en la educación de nuestros pueblos originarios:

\section{DISCURSO.}

La (M) se refiere al maestro:

M -buenos días niños, ¿cómo están? Contestan los alumnos. 
M -antes de venirse a la escuela ¿les dijeron gracias a sus padres por el alimento que les dieron hoy? contestan los alumnos.

M -Bueno si no lo hicieron vamos a hacerlo todos, pero ahora, hablándole a la tierra, como si fueran nuestros padres.

Discurso como propuesta. Desde la cosmovisión y cultura del pueblo de Santa Cruz Yagavila.

\begin{tabular}{|c|c|}
\hline LEN DIDZA XHIDZA (EN ZAPOTECO) & INTERPRETADO AL CASTEI \\
\hline $\begin{array}{l}\text { Padiusxh yuuyaadzaa kieto, xhkalenu benu } \\
\text { kieto tudo yiaj gakutu na'dza, xhkalenu } \\
\text { rhunu kietu lataj rleitu ben nii du ki yedzi lu } \\
\text { yuu, xhkalentiku waka, lii buniti loo netu, len } \\
\text { ben xhanlachhu, na'a zatu lataj kiu ni, tebe } \\
\text { zaatu ga guntu tu kebenaka, netu zaatu } \\
\text { rhenitu gakatu tu naka bën, nia ki tua na } \\
\text { rnabatu loo naku xhnatu yuuyaadzaa, ki ka } \\
\text { gaka lenu netu len icheo' netu ga galitu dza } \\
\text { chhia'a,lakaska rnabatu ki yuguteke bëni } \\
\text { gulunke yu'u skuela ni, bëni ni zuake len bëni } \\
\text { rdaa ni bë'gracia do kieke, laska ka be } \\
\text { bulezëdabe ni, bulekankabe kietu lata ni, chi } \\
\text { bueki dzayela bë’gracia kiakabe, rgin } \\
\text { ranabatu gakalenle netu yugutee tu rhuntu, } \\
\text { laska rnabatu loole lubi'le guka kiele lataj ni, } \\
\text { unitilole netu cha rhuntu tu kebe naka, cha } \\
\text { rhuleaj lachhi niatu ni, cha rbëchhiyatu, tebe } \\
\text { guni keile buleniti loo netu, laska li'i ka } \\
\text { xhnanu yuuyaazaa, bunitiloo netu len ben } \\
\text { xhanlachhu. Tebe dekas bila nuatu kiu, lii zii } \\
\text { xhënu, len zii } x h e ̈ n l e ~ t z k a ~ d u d u ~ r h u n a t u ~ k i u \\
\text { le kiele ¿waka?. }\end{array}$ & $\begin{array}{l}\text { Buenos días madre tierra nuestra, monte, día, } \\
\text { (yuuyaadzaa kieto) gracias por darnos el } \\
\text { alimento de hoy, gracias por darnos la } \\
\text { oportunidad de ver un nuevo día, muchas } \\
\text { gracias, tu piérdenos de vista, y has grande tu } \\
\text { corazón, hoy que hemos venido a este lugar, } \\
\text { no para hacer cosas malas sino para aprender } \\
\text { nuevas y buenas cosas, por eso te pedimos } \\
\text { permiso madre tierra, monte y día, para que } \\
\text { nos cuides y nos guíes, para así llegar en un } \\
\text { término feliz el día de hoy, de igual forma } \\
\text { pedimos por todos los señores que } \\
\text { construyeron esta escuela, los que viven y los } \\
\text { que con sus espíritus nos ven al igual que los } \\
\text { alumnos que ya fallecieron y nos dejaron todo } \\
\text { este espacio y que su espíritu se ha vuelto en } \\
\text { día y noche, les pedimos que nos cuiden en } \\
\text { cada una de las actividades que realicemos, } \\
\text { también pedimos a los dueños del lugar para } \\
\text { que nos pierdan de vista si hacemos cosas } \\
\text { malas o si pisoteamos este lugar, si gritamos } \\
\text { si jugamos, no nos hagan caso, piérdannos de } \\
\text { vista, igual a ti madre tierra piérdenos de vista } \\
\text { y haz grande tu corazón. } \\
\text { No tenemos más que darte y darles, pero } \\
\text { recibe y reciban esto que les ofrecemos ¿si?. }\end{array}$ \\
\hline
\end{tabular}

Después de este discurso, el maestro junto con los alumnos depositará sus ofrendas al lugar en donde se encuentren llevando a cabo la ceremonia; en la cultura zapoteca se le ofrece a la madre 
tierra productos como: cacao, aguardiente, caldo de pollo, pescado seco, granos de maíz, frijol. Acción que tiene como fin agradecer y respetar a los seres vivos y no vivos y a los espíritus del lugar donde se encuentren.

\section{Conclusiones}

Para concluir como aporte se presenta un modelo o un ejemplo de discurso donde se recalcan las palabras sabias de los ancianos, para que los docentes lo puedan adecuar en sus prácticas cotidianas y de acuerdo a las necesidades de los alumnos y al contexto en el que se encuentran. Todo esto se hará con el objetivo de visibilizar lo saberes de nuestros pueblos y de los niños, ya que ellos son portadores de estos conocimientos. Sin tener que hacerlo de manera forzosa u ocuparlo como puente para enseñar lo que en el plan y programa de estudio establece. Es ésta una de las formas que como investigador doy para que los docentes analicen las palabras para resignificar los saberes desde las aulas. Pero partiendo siempre del contexto de los alumnos para así visibilizar, enriquecer y potencializar los saberes comunitarios, elemento importante que aporta ésta investigación.

\section{Referencias}

BERTELY, M. Conociendo nuestras escuelas, un acercamiento etnográfico a 1 cultura escolar. México: Paidos, 2000.

FREIRE P. La educación como práctica de la libertad, Edit. Siglo XXI, México. 1997

FREIRE P. Política y Educción, 2 Edic. Edit. Siglo XXI, Mexico, Méx.1997

LEFF, E. Aventuras de la epistemología ambiental. De la articulación de las creencias al dialogo de saberes. México: Siglo XXI editores, 2006.

LÓPEZ L. E. Lengua y Educación. En Lengua II en Educación Bilingüe y Bicultural, vol.II, Capítulo VI. Santiago de Chile. UNESCO ORELAC. (1989)

MARÍN, Guillermo. Historia verdadera del México profundo. 1. ed. 1997. México: 2010.

MEYER, L. y MALDONADO, B. Comunalidad, educación y resistencia indígena en la era global. Un diálogo entre Noam Chomsky y más de 20 líderes e intelectuales del continente americano. México: SCIIO, 2011. 
OCAMPO L. J. Paulo Freire y la pedagogía del oprimido. Revista Historia de la Educación Latinoamericana, (10), 57-72.[Fecha de consulta 26 de Noviembre de 2020]. ISSN: 0122-7238. Disponible en: https://www.redalyc.org/articulo.oa?id=869/86901005 , 2008.

OLIVÉ L. (). Por una auténtica interculturalidad basada en el reconocimiento de la pluralidad epistemológico. CLASCO, CIDES- UMSA Muela de diablo Editores comuna, 2009. Recuperado de http://biblioteca.clasco/coediciones /20/60315033034/olivé.pdf.

ORTEGA P. La pedagogía crítica: Reflexiones en torno a sus prácticas y sus desafíos. Pedagogía y Saberes, No.31 2009 Recuperado de: file:///C:/Users/wilma/Downloads/659Texto\%20del\%20art\%C3\%ADculo-2346-1-10-20110928.pdf

PÉREZ G. R. La sierra Juárez, $1^{\circ}$ Edic. Instituto Oaxaqueño de las Culturas, Oaxaca, Oaxaca. 1956.

PÉREZ, A. [Berritzegune Nagusiak Antolatutaco Kilatatea Hezkuntzan jardunaldian] Prácticas educativas relevantes en la era digital, 2013. Recuperado en https:youtu.be/AKOludDPJSg.

RENGIFO G.V. Una Escuela Amable con el Saber Local, Proyecto andino de Tecnologías Campesinas $1^{\circ}$ Edic. PRECTEC. Perú. (2003)

RUIZ, B. Los huehuetlatolli: modelos discursivos destinados a la enseñanza retórica en la tradición indígena. p. 270-281. Universidad Miguel Hernández de elche, 2013.

WALSH C. Interculturalidad, plurinacionalidad y decolonialidad: las insurgencias políticoepistémicas de refundar el Estado, No.9: 131-152. Universidad Andina Simón Bolívar, Ecuador. Tabula Rasa. Bogotá - Colombia. 2008

WALSH C. Interculturalidad, plurinacionalidad y decolonialidad: las insurgencias políticoepistémicos de refundar el Estado. Tabula Rosa, núm. 9, Universidad Colegio Mayor de Cundinamarca, Colombia. 2008

WALSH C. Pedagogías decoloniales: Prácticas insurgentes de resistir, (re)existir y (re)vivir. TOMO II, Quito-Ecuador: Ediciones Abya-Yala, 2010.

Submetido em 17 de setembro de 2020.

Aceito em 09 de fevereiro de 2021

Publicado em 02 de março de 2021. 